\title{
Immunologic responses to the major allergen of Olea Europaea in local and systemic allergic rhinitis subjects
}

\author{
Paloma Campo ${ }^{1 *}$, Carmen Rondon², Mayte Villalba ${ }^{3}$, Esther Barrionuevo², Luisa Galindo², \\ Juan Carlos Lopez-Rodriguez ${ }^{3}$, Maria Jose Torres², Cristobalina Mayorga ${ }^{2}$, Miguel Blanca ${ }^{2}$ \\ From The 10th Symposium of Experimental Rhinology and Immunology of the Nose (SERIN 2015) \\ Stockholm, Sweden. 19-21 February 2015
}

\section{Background}

Ole e 1 is one of the major allergens from olive tree pollen. Up to date there are no specific studies that evaluate in depth the in vitro responses to this purified allergen. The goal of the study was to thoroughly evaluate the celullar responses to nOle e 1 in allergic rhinitis (AR) and local allergic rhinitis (LAR) patients with sensitization to olive tree pollen (OL) demonstrated by nasal allergen provocation test (NAPT).

\section{Methods}

Twelve subjects with AR (+NAPT with OL, + skin testing and specific IgE (sIgE) to OL), 12 subjects with LAR (+ NAPT with OL, - skin testing and SIgE to OL), and 12 subjects as control group (CG) (- NAPT, - skin testing and sIgE to OL) were selected. Basophil activation tests (BAT) with OL and nOle e 1, along with dendritic cell (DC) maturation/proliferation studies in response to nOle e 1 stimulation, were carried out in all subjects. Local ethical committee approved the study.

\section{Results}

All AR subjects had positive BAT responses to OL and 10/12 to nOle e1 (83\%); 8/12 LAR (66.6\%) had a positive BAT with OL and 4/12 (33\%) to nOle e1, with only one subject of the control group with a positive BAT to both $\mathrm{OL}$ and nOle e1 (8\%). DC proliferation and maturation were increased in $\mathrm{SAR}>\mathrm{LAR}>\mathrm{CG}$ but with no significant differences (maturation: 66.7\%/57\%/50\%; proliferation: $40 \% / 20 \% / 0 \%)$.

'Regional Hospital of Malaga, Malaga, Spain

Full list of author information is available at the end of the article

\section{Conclusion}

BAT with OL and nOle e1 in LAR group showed sensitivity between 66.6 and 33\%, demonstrating specific basophil activation with pollens in patients with LAR. DC proliferation and maturation were demonstrated in SAR and LAR subjects although with no significant differences with CG.

\section{Authors' details}

'Regional Hospital of Malaga, Malaga, Spain. ${ }^{2}$ Regional Hospital of Malaga, UGC Allergy, Malaga, Spain. ${ }^{3}$ Faculty of Chemistry, Complutense University of Madrid, Department of Biochemistry and Molecular Biology, Madrid, Spain.

Published: 26 June 2015

doi:10.1186/2045-7022-5-S4-P19

Cite this article as: Campo et al:: Immunologic responses to the major allergen of Olea Europaea in local and systemic allergic rhinitis subjects. Clinical and Translational Allergy 2015 5(Suppl 4):P19.
Submit your next manuscript to BioMed Central and take full advantage of:

- Convenient online submission

- Thorough peer review

- No space constraints or color figure charges

- Immediate publication on acceptance

- Inclusion in PubMed, CAS, Scopus and Google Scholar

- Research which is freely available for redistribution

Submit your manuscript at www.biomedcentral.com/submit
() Biomed Central 